\title{
ELASTIC INTERACTION ENERGY OF POINT DEFECTS WITH A BASAL DISLOCATION LOOP IN ZIRCONIUM
}

\author{
O.G. Trotsenko, A.V. Babich \\ Institute of Electrophysics and Radiation Technologies NAS of Ukraine, Kharkiv, Ukraine \\ E-mail: oleg-trotsenko@ukr.net
}

Based on the tensor Green's function for hexagonal crystals obtained by the Lifshitz-Rosenzweig method, the analytical expression of the elastic interaction energy of radiation point defects is calculated in the model of force dipoles with a dislocation vacancy loop (basal plane of occurrence $\{0001\}$, Burgers vector $\mathbf{b}^{\mathbf{D S}}=1 / 6\langle 20 \overline{2} 3\rangle$ ), in zirconium.

PACS: 62.20.Dc; 62.20.Fe

\section{INRODUCTION}

Under irradiation with high-energy particles, point defects (vacancies and interstitial atoms (SIA)) are generated in the crystal. The future of these defects is diffusion migration and absorption by macroscopic sinks (pores, dislocations, grain boundaries, etc.) or recombination upon encounter. As a result, the initial crystal structure changes, which leads to such phenomena as radiation swelling, radiation growth, and other phenomena. During irradiation zirconium alloys behave differently from most other engineering alloys in that they resist swelling. They do exhibit anisotropic dimensional changes in the absence of an applied stress that depend on the microstructure; this process is called irradiation growth. Straight-line dislocations and dislocation loops are important internal sinks. And knowledge of the processes that control their evolution is very important for understanding the mechanisms of radiation damage.

According to the generally accepted concept (the socalled bias factor) the edge straight-line dislocations, and the dislocation loops absorb SIA better than vacancies. The reason is a stronger attraction of the SIA due to the elastic interaction. Moreover, its value does not depend on the nature of the loop (interstitial or vacancy). Therefore, according to the ideology, there should not be vacancy loops on a macroscopic scale. For BCC and GCC metals, this point is acceptable (they demonstrate radiation swelling) [1]. In particular, for steels, this conclusion is confirmed by numerous experiments [2].

As for HCP metals, along with interstitial vacancies in there vacancy loops of rather large sizes are also observed under irradiation [3]. The phenomenon of radiation growth, in particular, in zirconium, is associated with them. Radiation growth is accompanied by a change of the shape of the material without the application of an external load and a noticeable change in volume. Thus zirconium during radiation growth expands along the $\langle\mathrm{a}\rangle$-direction and narrows along the $\langle\mathrm{c}\rangle$-axis [4]. One of the variations in its shape is explained by the growth of vacancy loops in the basal planes, and interstitial - in prismatic ones. Today, there is no complete understanding of the existence of loops of both types in HCP metals. The most common version of such a distribution of point defect (PD) flows is anisotropic diffusion between zirconium planes [5]. However, it does not explain the dependence on the radius of the loop, its nature, and other characteristics. Perhaps the elastic anisotropy of HCP metals can explain the phenomenon of radiation growth. It is considered in the work that it is too early to discard the elastic interaction. It is necessary to calculate it correctly. It is possible that dislocation loops on different planes of the HCP metal have an elastic preference factor different from the magnitude of the preference for SMA. Then, under irradiation, SIAs will be better absorbed by loops with a large bias factor. Vacancies that remain in excess will go on loops with a smaller bias factor. And, if the formers are on prismatic planes, and the others on the basal planes, then there is a place for the separation of flows between the planes, but purely elastic.

The goal of the work is to apply the expressions obtained earlier based on tensor Green functions for hexagonal crystals in the Lifshitz-Rosenzweig approach to calculate the elastic energy of radiation interaction of radiation point defects with a vacancy basis loop with a Burgers vector $\mathbf{b}^{\mathbf{D S}}=1 / 6\langle 20 \overline{2} 3\rangle$ in zirconium.

\section{BASIC EQUATIONS}

In the paper, the classical formula [6, 7] was used for the displacement due to dislocation in an anisotropic medium, which is modeled by the tensor Green's function:

$$
u_{i}(\vec{r})=C_{j k l m} b_{m} \int_{S_{D}} n_{l} G_{i j, k}\left(\vec{r}-\vec{r}^{\prime}\right) d^{2} r^{\prime} ; \quad G_{i j, k} \equiv \frac{\partial}{\partial x_{k}} G_{i j}
$$

Here $C_{j k l m}$ - tensor of elastic moduli of a medium simulating a crystal; $b_{m}$ - component of the Burgers vector of the dislocation; $n_{l}$-component of the normal vector to an arbitrary surface $S_{D}$, based on a dislocation line $D ; G_{i j}$ - tensor Green function; $\vec{r}$ - point of observation; surface integration $S_{D} ; \vec{r}^{\prime}$ - surface point coordinate.

The energy of interaction between two systems of internal stresses $S\left(\boldsymbol{u}^{S}, e_{i j}^{S}, \sigma_{i j}^{S}\right)$ and $T\left(\boldsymbol{u}^{T}, e_{i j}^{T}, \sigma_{i j}^{T}\right)$, with a known fictitious distribution of volume forces, which simulates a real source $S$ according to Eshelby [8], can be represented by an integral of the form:

$$
E_{\text {int }}(S, T)=-\int f_{i}^{S} u_{i}^{T} d V,
$$

which is taken over an area containing only the source of the system $S$. Let this system be caused by a PD, described in the theory of elasticity by the volume distribution of dipole forces without moment 


$$
f_{i}\left(\boldsymbol{r}-\boldsymbol{r}_{0}\right)=-P_{i j} \nabla_{j} \delta\left(\boldsymbol{r}-\boldsymbol{r}_{0}\right) ; \quad P_{i j}=P_{j i} .
$$

For the interaction energy between it and the stress system $T$, we have

$$
E_{\mathrm{int}}(\boldsymbol{r})=-\int f_{i}\left(\boldsymbol{r}^{\prime}-\boldsymbol{r}\right) u_{i}^{T}\left(\boldsymbol{r}^{\prime}\right) d \boldsymbol{r}^{\prime}=-P_{i j} e_{i j}^{T}(\boldsymbol{r}) .
$$

Here $P_{i j}$ - power of dipole moments.

For a hexagonal crystal, the power components of dipole moments $P_{i j}$ supposed in the form $P_{11}=P_{22}=P$;

$$
P_{33} \neq P_{11} ; \quad P_{12}=P_{13}=P_{23}=0 \text {. Let } \varepsilon=\frac{P_{33}}{P_{11}} \text {. }
$$

Substituting the power of dipole moments into formula (4) $P_{i j}$ and components of the strain tensor $e_{i j}$ [9] we have the following expression for the interaction energy between a dislocation loop and a point defect:

$$
E_{\mathrm{int}}^{D S} \equiv(-1)\left(P_{11} e_{11}^{D S}+P_{22} e_{22}^{D S}+P_{33} e_{33}^{D S}\right)=(-1)\left(P_{11}\left(e_{11}^{D S}+e_{22}^{D S}\right)+\varepsilon P_{11} e_{33}^{D S}\right)=-P_{11}\left(\left(e_{11}^{D S}+e_{22}^{D S}\right)+\varepsilon e_{33}^{D S}\right) .
$$

Here $e_{i i}^{D S}(\boldsymbol{r})=e_{i i}^{D}(\boldsymbol{r})+e_{i i}^{S}(\boldsymbol{r})$ represented by the sum of the strain tensors due to the contribution of the shear $e_{i i}^{S}$ and prismatic $e_{i i}^{D}$ loop components

$$
\begin{aligned}
& e_{i i}^{D}(\boldsymbol{r})=b^{D} \int_{S_{D}} d^{2} r^{\prime}\left[C_{13} \sum_{\alpha=1}^{2} G_{i \alpha, \alpha i}\left(\boldsymbol{r}-\boldsymbol{r}^{\prime}\right)+C_{33} G_{i 3,3 i}\left(\boldsymbol{r}-\boldsymbol{r}^{\prime}\right)\right], \\
& e_{i i}^{S}(\boldsymbol{r})=b^{S} C_{44} \int_{S_{D}}\left[G_{i 3, i 1}\left(\boldsymbol{r}-\boldsymbol{r}^{\prime}\right)+G_{i 1, i 3}\left(\boldsymbol{r}-\boldsymbol{r}^{\prime}\right)\right] d^{2} r^{\prime} .
\end{aligned}
$$

The paper considers a circular vacancy loop of radius $R$, which lies on the plane $z=0$. An example of an object of this kind is $c$ - loops for the Burgers vector $\mathbf{b}^{\mathbf{D S}}=1 / 6\langle 20 \overline{2} 3\rangle$ in zirconium under neutron irradiation at a temperature from 560 to $773 \mathrm{~K}$ [10]. The Burgers vector is at an angle to the normal vector $x$ axis - along the direction of the projection of the Burgers vector in the basal plane, $z$ - along the normal vector.

Expand the Burgers vector $\mathbf{b}^{\mathrm{DS}}=1 / 6\langle 20 \overline{2} 3\rangle$ on prismatic $\mathbf{b}^{\mathbf{D}}=1 / 2\langle 0001\rangle$ (perpendicular to the loop plane) and shear $\mathbf{b}^{\mathbf{S}}=1 / 3\langle 10 \overline{1} 0\rangle$ (lying in the loop plane) components. Their modules correspond to the components $b^{D S}, b^{D}, b^{S}$. Using the inverse transition formulas from the Miller-Brave indices to the Miller indices

$$
u=\frac{2 r+s}{3} ; v=\frac{r+2 s}{3} ; w=\frac{n}{3},
$$

we get the vectors in 3 dimensional coordinates:

$\mathbf{b}^{\mathbf{D S}}=1 / 18\langle 423\rangle ; \mathbf{b}^{\mathbf{S}}=1 / 9\langle 210\rangle ; \mathbf{b}^{\mathbf{D}}=1 / 6\langle 001\rangle$.

Modulus of vector $b^{D}=1 / 6$. Since the angle between the axis in the HCP lattice is 120 degrees, modulus of vector $b^{S}=\sqrt{12} / 18$. Burger's vector $\mathbf{b}^{\mathbf{D S}}$ module will be equal $b^{D S}=\sqrt{\left(b^{D}\right)^{2}+\left(b^{S}\right)^{2}}=\sqrt{21} / 18$.

The tensor of strain $e_{i i}^{D S}(\boldsymbol{r})$ (6) presented (5) by the integral in the form:

$$
E_{\mathrm{int}}^{D S} \equiv-P_{11} \int_{S_{D}} d^{2} r^{\prime}\left(C_{44} b^{S}\left[\begin{array}{l}
\sum_{\alpha=1}^{2} G_{\alpha 3, \alpha 1}+\sum_{\alpha=1}^{2} G_{\alpha 1, \alpha 3}+ \\
+\varepsilon\left[G_{33,13}\left(\boldsymbol{r}-\boldsymbol{r}^{\prime}\right)+G_{31,33}\left(\boldsymbol{r}-\boldsymbol{r}^{\prime}\right)\right]
\end{array}\right]+b^{D}\left[\begin{array}{l}
C_{13} \sum_{\gamma=1}^{2} \sum_{\alpha=1}^{2} G_{\gamma \alpha, \alpha \gamma}+\varepsilon C_{33} G_{33,33}\left(\boldsymbol{r}-\boldsymbol{r}^{\prime}\right) \\
+\left(C_{33}+\varepsilon C_{13}\right) \sum_{\alpha=1}^{2} G_{3 \alpha, \alpha 3}\left(\boldsymbol{r}-\boldsymbol{r}^{\prime}\right)
\end{array}\right]\right) .
$$

Let's write the components: $\varepsilon$ and $P_{11}$ for hexagonal crystal through dilation volume $\Delta V$ and coefficient $\gamma_{Q}$, which are determined experimentally. To do this, we will rewrite these components through the displacement dipole. For hexagonal crystal, the displacement dipole are presented in this form $Q_{11}=Q_{22} ; Q_{33} \neq Q_{11} ; Q_{12}=Q_{13}=Q_{23}=0$, where is $\gamma_{Q}=Q_{33} / Q$. Because $P_{i j}=C_{i j k l} Q_{k l}$, then

$$
\begin{aligned}
& P_{11}=P=\left(C_{11}+C_{12}+\gamma_{Q} C_{13}\right) Q_{11}, \\
& \varepsilon\left(\gamma_{Q}\right)=\frac{2 C_{13}+\gamma_{Q} C_{33}}{C_{11}+C_{12}+\gamma_{Q} C_{13}} .
\end{aligned}
$$

Consider the forces acting on the point defect in the hexagonal crystal, the balance of power equation $\sigma_{i j, j}+f_{i}=0$. Here $f_{i}=-C_{i j l} Q_{l l} \nabla_{j} \delta(\vec{r}) \quad$ and $\sigma_{i j}=C_{i j l} e_{l l}$ for a point defect, the sum of diagonal elements of the strain tensor can be presented through dilation volume $e_{l l}=\operatorname{div} \vec{u}=\Delta V \delta(\vec{r})$. Then, by substituting $f_{i}$ and $e_{l l}$ in the equation of equilibrium of forces that act on a point defect, we have $C_{i j l l} \nabla_{j} \Delta V \delta(\vec{r})-C_{i j l l} Q_{l l} \nabla_{j} \delta(\vec{r})=0$ or $Q_{11}=\Delta V /\left(2+\gamma_{Q}\right)$. Putting in the equation (10) we have components: $\varepsilon$ and $P_{11}$, scheduled through experimentally obtained data, such as dilatation volume and coefficient $\gamma_{Q}$ :

$$
\begin{aligned}
& P_{11}=P=\frac{\left(C_{11}+C_{12}+\gamma_{Q} C_{13}\right)}{\left(2+\gamma_{Q}\right)} \Delta V, \\
& \varepsilon\left(\gamma_{Q}\right)=\frac{2 C_{13}+\gamma_{Q} C_{33}}{C_{11}+C_{12}+\gamma_{Q} C_{13}} .
\end{aligned}
$$

\section{THE GREEN'S FUNCTIONS METHOD: LIFSHITZ-ROSENZWEIG'S APPROACH}

The formula (9) takes the following form [9] according to the Lifshitz-Rosenzweig approach: 


$$
E_{\mathrm{int}}^{D S}(\vec{\rho}) \equiv-P \frac{1}{R} \int_{S_{D}} \frac{d^{2} \rho^{\prime}}{\left|\rho-\rho^{\prime}\right|^{3}}\left(b^{S} \tau_{3} \tau_{1} \mathrm{H}^{S}\left(\tau_{3}^{2}\right)-b^{D} \mathrm{H}^{D}\left(\tau_{3}^{2}\right)\right)
$$

where

$$
\begin{aligned}
& \mathrm{H}^{S}\left(\tau_{3}^{2}\right)=\left[3\left\{\Psi\left(\tau_{3}^{2}\right)-Y\left(\tau_{3}^{2}\right)\right\}+2 \tau_{3}^{2} \frac{d \Psi}{d \tau_{3}^{2}}+2\left(1-\tau_{3}^{2}\right) \frac{d Y}{d \tau_{3}^{2}}\right], \\
& \mathrm{H}^{D}\left(\tau_{3}^{2}\right)=\left[\left(1-3 \tau_{3}^{2}\right) Q\left(\tau_{3}^{2}\right)+3 \tau_{3}^{2}\left(1-\tau_{3}^{2}\right) \frac{d Q}{d \tau_{3}^{2}}\right] .
\end{aligned}
$$

The result ispresented in dimensionless cylinrical coordinates $\rho=r / R, \varphi, \zeta=z / R$. The notation here is as follow:

$$
\begin{aligned}
& \tau_{1}=\left(\rho \cos (\varphi)-\rho^{\prime} \cos \left(\varphi^{\prime}\right)\right) /\left|\rho-\rho^{\prime}\right| ; \\
& \tau_{2}=\left(\rho \sin (\varphi)-\rho^{\prime} \sin \left(\varphi^{\prime}\right)\right) /\left|\rho-\rho^{\prime}\right| ; \\
& \tau_{3}^{2}=\zeta^{2} /\left|\rho-\rho^{\prime}\right|^{2} ; \\
& \left|\rho-\rho^{\prime}\right|^{2}=\rho^{2}+\zeta^{2}-2 \rho \rho^{\prime} \cos \left(\varphi-\varphi^{\prime}\right)+\rho^{\prime 2} ; \\
& \varphi, \varphi^{\prime}-\text { azimuth angles. } \\
& Q\left(\tau_{3}^{2}\right)=C_{13} \mathrm{~K}\left(\tau_{3}^{2}\right)+\varepsilon C_{33} W\left(\tau_{3}^{2}\right)+\left(\varepsilon C_{13}+C_{33}\right) V\left(\tau_{3}^{2}\right) ; \\
& \Psi\left(\tau_{3}^{2}\right)=C_{44}\left(V\left(\tau_{3}^{2}\right)+\varepsilon W\left(\tau_{3}^{2}\right)\right) ; \\
& Y\left(\tau_{3}^{2}\right)=C_{44}\left(\mathrm{~K}\left(\tau_{3}^{2}\right)+\varepsilon V\left(\tau_{3}^{2}\right)\right) .
\end{aligned}
$$

Functions $\mathrm{K}\left(\tau_{3}^{2}\right), W\left(\tau_{3}^{2}\right), V\left(\tau_{3}^{2}\right)$ are very complicated and therefore made in the Appendix.

The result is given as the sum of two loop components:

edge

$$
E_{\text {int }}^{D}(\vec{\rho}) \equiv P \frac{1}{R} \int_{S_{D}} \frac{d^{2} \rho^{\prime}}{\left|\rho-\rho^{\prime}\right|^{3}}\left(b^{D} \mathrm{H}^{D}\left(\tau_{3}^{2}\right)\right)
$$

and shear

$$
E_{\text {int }}^{S}(\vec{\rho}) \equiv-P \frac{1}{R} \int_{S_{D}} \frac{d^{2} \rho^{\prime}}{\left|\rho-\rho^{\prime}\right|^{3}}\left(b^{S} \tau_{3} \tau_{1} \mathrm{H}^{S}\left(\tau_{3}^{2}\right)\right) .
$$

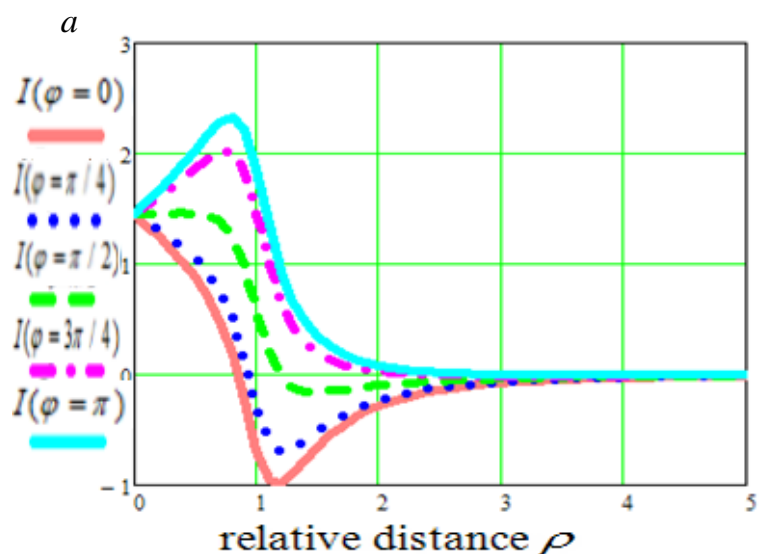

At the edge, the Burgers vector is directed along the $\mathrm{z}$ axis. For this part, there is cylindrical symmetry with respect to rotation. She has no azimuthal dependence. For the shear part, the Burgers vector is directed parallel to the basal plane. This result depends on the azimuthal angle.

\section{RESULTS}

Numerical evaluations have been carried out for zirconium. The experimental values of its elastic moduli according to [11] are as follows (Mbar): $C_{11}=1,554$; $C_{12}=0,672 ; C_{13}=0,646 ; C_{33}=1,725 ; C_{55}=C_{44}=0,363$. The comparison results are presented in Figs. 1-5 in dimensionless cylindrical coordinates $\rho=r / R ; \varphi$; $\zeta=z / R$.

1. First, consider the center of dilatation, when $\varepsilon=1$

Fig. 1 shows the radial dependence for the dimensionless interaction energy $I(\rho, \varphi, \zeta)=-R E_{\text {int }}^{D S} / b^{D S} P$ of the dislocation loop and PD for the planes $z=0.1 R$ and $z=0.5 R$, where $\zeta=z / r$. Functions with angles $0, \pi / 4, \pi / 2,3 \pi / 4$, $\pi$ are shown in Fig. 1.

Dependency in range $\pi-2 \pi$ is the same as in the opposite direction. With increasing angle, the minimum in the radial dependence decreases. In range $0-\pi$ minimum transforms to maximum.

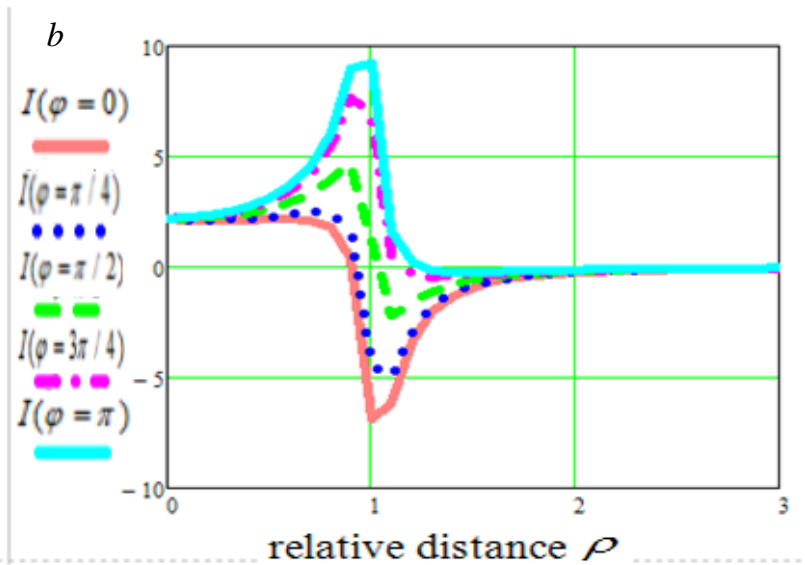

Fig. 1. Function $I(\rho, \varphi, \zeta)$ depending on the relative distance $\rho$ in the plane: $a-z=0.5 R$ and $b-z=0.1 R$

On Fig. 1 radial dependence on the angle $\pi / 2$ and $3 \pi / 2$ responds to the lack of contribution of the shift part. It for this angle qualitatively corresponds to the radial dependence of the vacancy edge loop (with the Burger's vector, $\mathbf{b}^{\mathbf{D}}=1 / 2\langle 0001\rangle$ ) in the base plane. Therefore, negative values show the area of attraction of vacancies, and positive - SIA; the area of repulsion is the opposite.

For corners from $\pi / 2$ to $3 \pi / 2$ the contribution is positive. Therefore, on the graphs of radial dependencies $3 \pi / 4, \pi$ you can see the greater attraction of the SMA. For angles $-\pi / 2$ from up to 
$\pi / 2$ in this range, the shift part gives a negative contribution, so on the graphs of radial dependencies 0 , $\pi / 4$ a greater attraction of vacancies than SIA except for a certain area in the middle of the loop. This is because the prismatic component of the interaction energy is more than a shift in the middle of the loop.

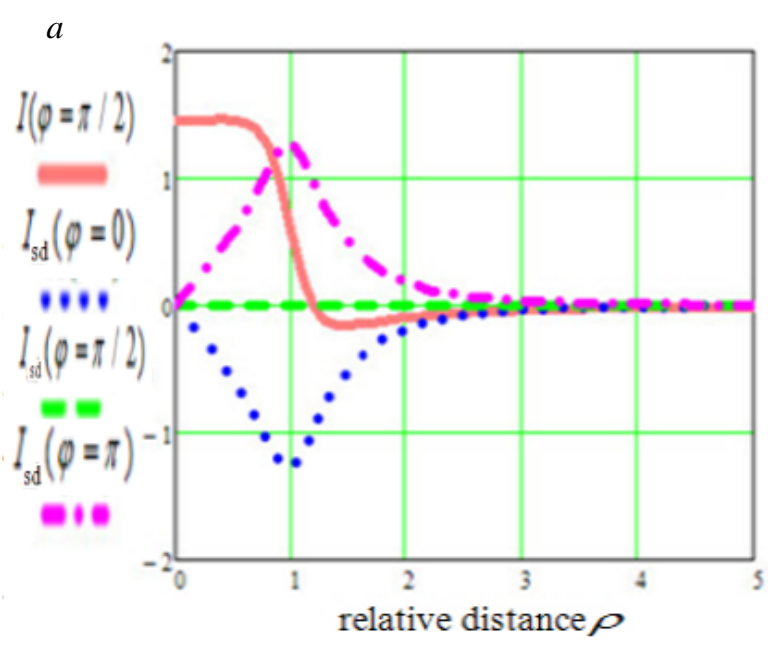

The shear energy of the interaction gives a negative contribution to the area $\left[0, \frac{\pi}{2}\right]$ and $\left[\frac{3 \pi}{2}, 2 \pi\right]$, and the positive in the field $\left[\frac{\pi}{2}, \frac{3 \pi}{2}\right]$.

$b$

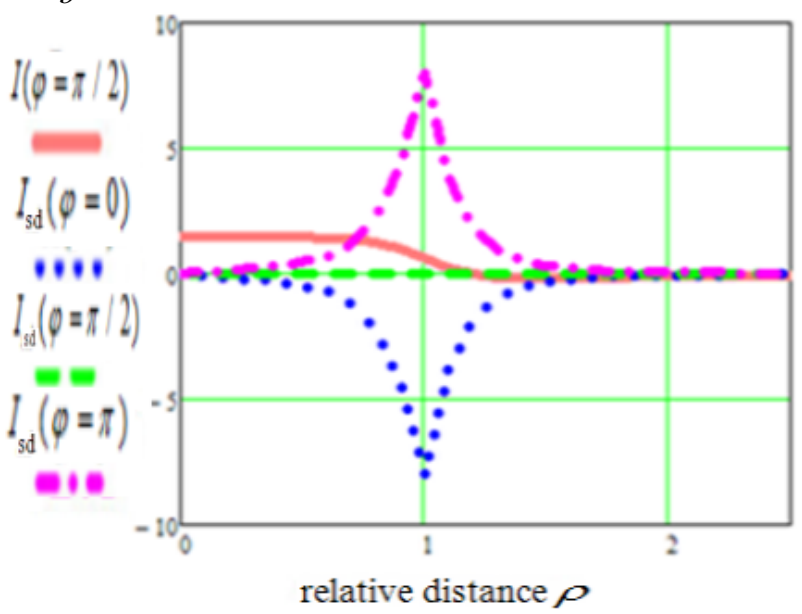

Fig. 2. Function $I(\rho, \varphi=\pi / 2, \zeta)$ and $I_{s d}(\rho,(\varphi=0, \pi / 2, \pi), \zeta)$ depending on the relative distance $\rho$ in the plane: $a-z=0.5 R$ and $b-z=0.1 R$

This contribution of the shear part $I(\rho, \varphi, \zeta)$ is shown in Fig. 2 versus the edge of the function $I(\rho, \varphi, \zeta)$, in the form of radial function dependence $I(\rho, \varphi=\pi / 2, \zeta)$, shear part of the function $I_{s d}(\rho, \varphi, \zeta)$, for angles $\varphi=0, \pi / 2, \pi . \quad \varphi=0$ corresponds to the maximum negative contribution of the shear part, $\varphi=\pi / 2$ the lack of contribution, $\varphi=\pi$ the maximum positive contribution.

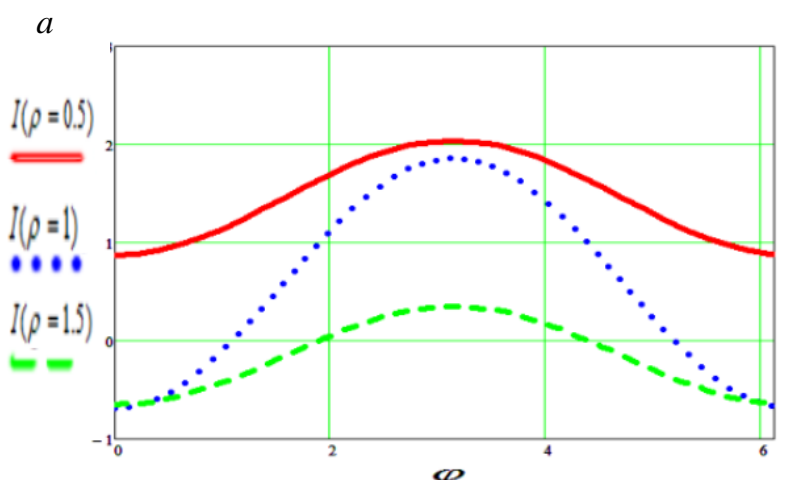

On Fig. 2 it is shown that near the loop the contribution of the shear part is greater than that of the radial part. When one move away from the loop the contribution of the edge part on the contrary becomes more than a shear one. The contribution of the shear part is concentrated near the dislocation line, so in the area inside the loop to a certain value of a disproportionate radius $\rho$, where the contribution of the shear part becomes commensurate with the edge, there is only the attraction of the SIA and repulsion vacancies.

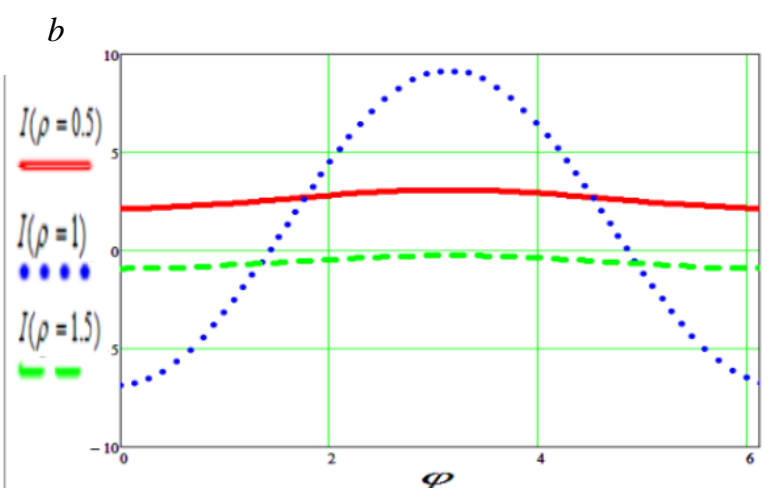

Fig. 3. Function $I((\rho=0.5,1,1.5), \varphi, \zeta)$ depending on the azimuthal angle in the plane: $a-z=0.5 R$ and $b-z=0.1 R$

The following Fig. 3 show exactly how the shear part contributes for two cases respectively, inside the loop area $0.5 R$ and beyond $1.5 R$. So for a $0.5 R$ radius we have a change in energy of interaction values only in a positive area. Outside, the same loop given angular dependence for a radius of $1.5 R$ as opposed to the area inside the loop has a case of changing the nature of the interaction to the opposite. In addition, the total area of attraction of vacancies is greater than the attraction of the ISA.
2. For a case $\varepsilon=P_{33} / P_{11} \neq 1$, when, the radial dependence for the immeasurable energy of interaction $I(\rho, \varphi, \zeta)=-R E_{\text {int }}^{D S} / b^{D S} P$ of the dislocation loop and point defect for planes $z=0.1 R$ and $z=0.5 R$, will be a little different. Consider three radial dependencies for: a) 0 ; b) $\pi / 2$; c) $\pi$ angles. For them, we will consider three values $\varepsilon=0.5,1,1.5$. The graphs show a flattened PD point line $\varepsilon=0.5$, a solid line dilation center $\varepsilon=1$, a stretched PD dotted line $\varepsilon=1.5$. 
The plane $z=0.5 R$ (Fig. 4)
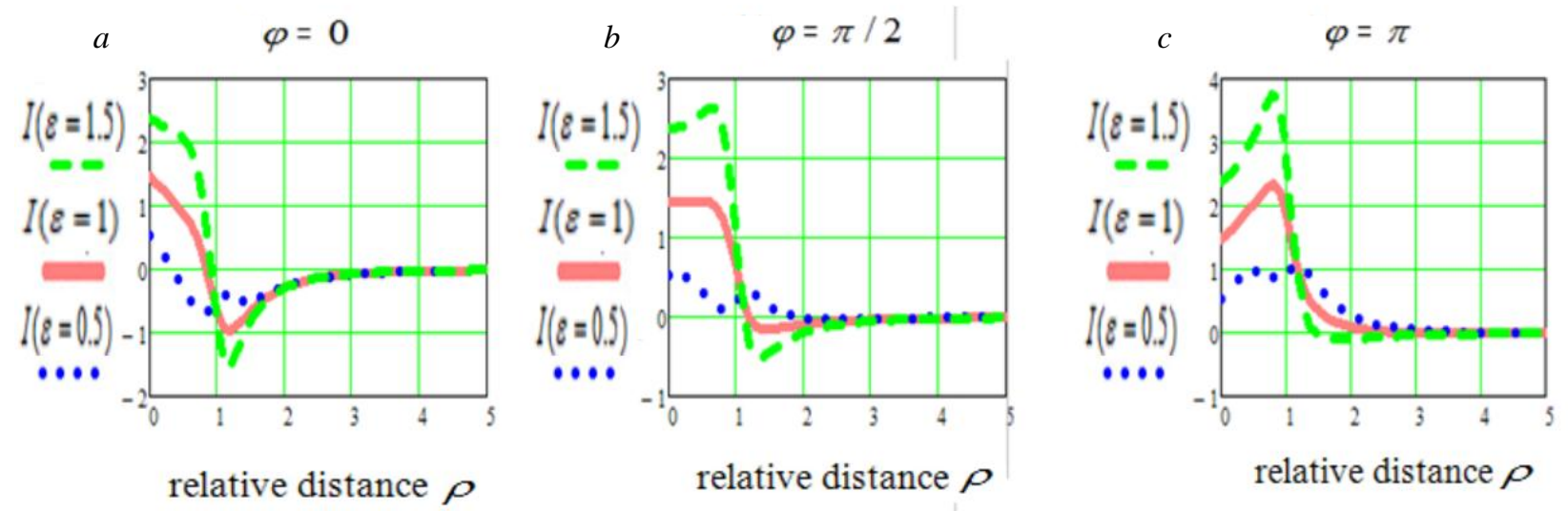

Fig. 4. Function $I(\rho, \varphi, \zeta)$ depending on the relative distance $\rho$ in the plane $z=0.5 R$ :

$$
a-\varphi=0 ; b-\varphi=\pi / 2 \text {, and } c-\varphi=\pi
$$

The plane $z=0.1 R$ (Fig. 5)
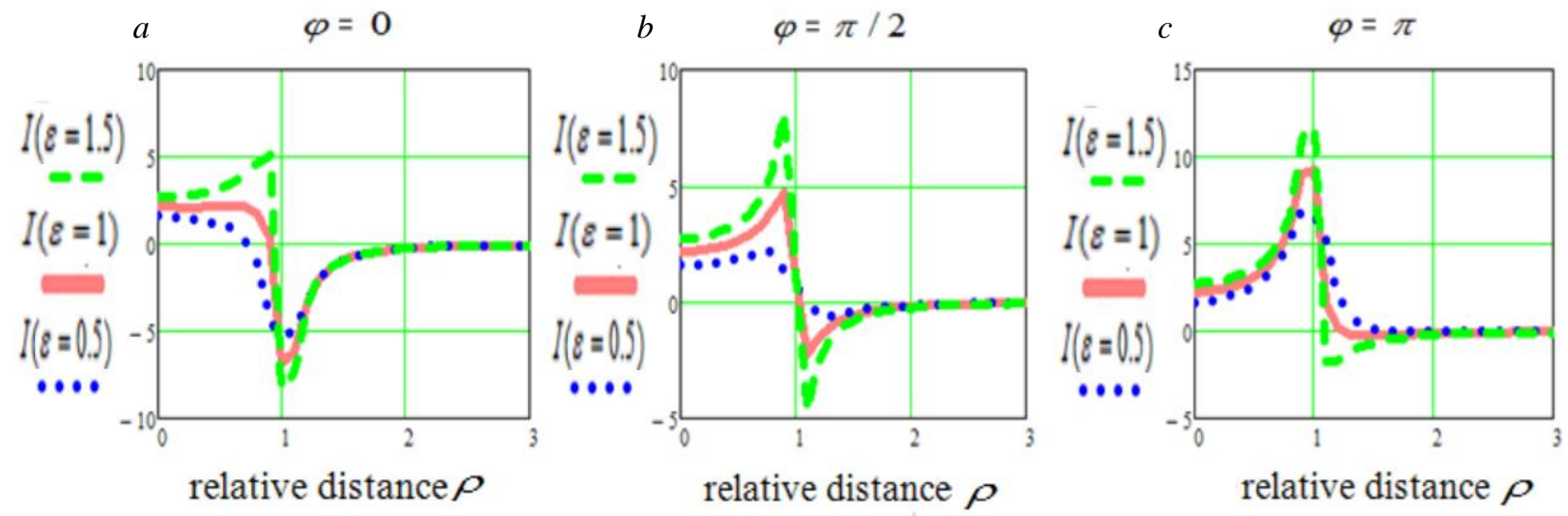

Fig. 5. Function $I(\rho, \varphi, \zeta)$ depending on the relative distance $\rho$ in the plane $z=0.1 R$ :

$$
a-\varphi=0 ; b-\varphi=\pi / 2 \text {, and } c-\varphi=\pi
$$

It can be noticed that more elongated point defects have an interaction energy much greater than at equal values $P(\varepsilon=1)$. Flattened point defects are less. The nature of the interaction corresponds to the center of dilation for the plane $z=0.5 R$.

However, the radial dependences for the $z=0.5 R$ plane have two minima for the angle 0 , with a subsequent transition to one minimum and one maximum for the angle $\pi / 2$ and up to two maxima for the angle $\pi$. This can be explained by the stronger contribution of the shear part to the radial dependence of the oblate point defect.

In addition, calculating from formula (11) $P$, and $\varepsilon$, it is possible to determine what type a point defect has, and also find the energy of elastic interaction.

\section{CONCLUSION}

The energy of elastic interaction for the real loop in the zirconium (basal plane of the lie, the Burger's vector $\mathbf{b}^{\text {DS }}=1 / 6\langle 20 \overline{2} 3\rangle$ ) with a point defect using the received in the [12] Green's tensor function method Lifhitz-Rosenzweig is calculated [13]. The result makes it possible to calculate diffusion fluxes of PD on a loop and its bias-factor and are important for study of such phenomenon as radiation growth in reactor materials under irradiation [14].

The authors are grateful to P.N. Ostapchuk for productive discussion of results.

\section{REFERENCES}

1. В.Ф. Зеленский, И.М. Неклюдов, Т.П. Черняева. Радиационные дефекты $u$ распухание металлов. Киев: «Наукова думка», 1988, 296 с.

2. F.A. Garner, M.B. Toloczko, B.H. Sencer. Comparison of swelling and irradiation creep behavior of fcc-austenitic and bcc-ferritic/martensitic alloys at high neutron exposure // J. Nucl. Mater. 2000, v. 276, p. 123.

3. C.H. Woo, U. Gosele. Dislocation bias in an anisotropic diffusive medium and irradiation growth // J. Nucl. Mater. 1983, v. 119, p. 219-228.

4. Sang Il Choi, Ji Hyun Kim. Radiation-induced dislocation and growth behavior of zirconium and zirconium alloys - a review // Nuclear Engineering and Technology. 2013, v. 45, N 3, p. 385-392.

5. C.H. Woo. Defect accumulation behaviour in hcp metals and alloys // J. Nucl. Mater. 2000, v. 276, p. $90-103$. 
6. Л.Д. Ландау, Е.М. Лифшиц. Теоретическая физика. T. VII. Теория упругости. М.: «Наука», 1987, $248 \mathrm{c}$.

7. Дж. Хирт, И. Лоте. Теория дислокащий. М.: «Атомиздат», 1972, 599 с.

8. Дж. Эшелби. Континуальная теория дислокаций. М.: «Наука», 1963, 215 с.

9. О.Г. Троценко, П.Н. Остапчук. Методы расчета упругого взаимодействия точечных дефектов с дислокационными петлями в гексагональных кристаллах // BAHT. 2017, №2(108), c. 83-90.

10. M. Griffiths. A review of microstructure evolution in zirconium alloys during irradiation // $\mathrm{J}$. Nucl. Mater. 1988, v. 159, p. 190-218.
11. L. Fast, J.M. Wills, B. Johansson, O. Eriksson. Elastic constants of hexagonal transition metals: Theory // Phys. Rev. 1995, v. B 51, p. 17431.

12. П.Н. Остапчук, О.Г. Троценко. Тензорная функция Грина в теории упругости анизотропной гексагональной среды // ВАНT. 2014, №4(92), с. 4954.

13. И.М. Лифшиц, Л.Н. Розенцвейг. О построении тензора Грина для основного уравнения теории упругости в случае неограниченной упругоанизотропной среды // ЖЭТФ. 1947, т. 17, с. 783791.

14. V.I. Dubinko, S.A. Kotrechko, V.F. Klepikov. Irradiation hardening of reactor pressure vessel steels due to the dislocation loop evolution // Radiation Effects and Defects in Solids. 2009, v. 164, p. 647.

\section{APPENDIX}

$$
\begin{aligned}
& V\left(\tau_{3}^{2}\right) \equiv\left(1-3 \tau_{3}^{2}\right) \Phi\left(\tau_{3}^{2}\right)+2 \tau_{3}^{2}\left(1-\tau_{3}^{2}\right) \frac{d \Phi}{d \tau_{3}^{2}} ; \\
& W\left(\tau_{3}^{2}\right) \equiv F\left(\tau_{3}^{2}\right)-2\left(1-\tau_{3}^{2}\right) \frac{d F}{d \tau_{3}^{2}} ; \\
& \mathrm{K}\left(\tau_{3}^{2}\right) \equiv-\mathrm{N}\left(\tau_{3}^{2}\right)-2 \tau_{3}^{2} \frac{d \mathrm{~N}}{d \tau_{3}^{2}}-3 \tau_{3}^{2} \mathrm{M}\left(\tau_{3}^{2}\right)+2 \tau_{3}^{2}\left(1-\tau_{3}^{2}\right) \frac{d \mathrm{M}}{d \tau_{3}^{2}} \Phi\left(\tau_{3}^{2}\right) \equiv \frac{2 i}{\left(z_{1}+z_{2}\right)} \frac{a+b+\chi+\rho}{A\left(\tau_{3}^{2}\right)} ; \\
& F\left(\tau_{3}^{2}\right) \equiv \frac{2 i}{\left(z_{1}+z_{2}\right) A\left(\tau_{3}^{2}\right)}\left((b+\rho)+(a+b-\rho) \tau_{3}^{2}-\frac{a+2 b}{z_{1} z_{2}}\right) ; \\
& \mathrm{N}\left(\tau_{3}^{2}\right) \equiv \frac{2 i}{\left(z_{1}+z_{2}\right)} \frac{R\left(\tau_{3}^{2}\right)}{b A\left(\tau_{3}^{2}\right)}-\frac{b \tau_{3}^{2}}{\sqrt{b P\left(\tau_{3}^{2}\right)}(b+\rho)\left(1-\tau_{3}^{2}\right)} ; \\
& \mathrm{M}\left(\tau_{3}^{2}\right) \equiv \frac{2 i}{\left(z_{1}+z_{2}\right)} \frac{S\left(\tau_{3}^{2}\right)}{\left(1-\tau_{3}^{2}\right) b A\left(\tau_{3}^{2}\right)}-\frac{P\left(\tau_{3}^{2}\right)+b \tau_{3}^{2}}{\sqrt{b P\left(\tau_{3}^{2}\right)}(b+\rho)\left(1-\tau_{3}^{2}\right)^{2}} ; \\
& R\left(\tau_{3}^{2}\right)=\frac{(a+b)(b+\rho)}{z_{1} z_{2}}-\frac{A\left(\tau_{3}^{2}\right) P\left(\tau_{3}^{2}\right)}{2(b+\rho)\left(1-\tau_{3}^{2}\right)}\left(z_{1} z_{2}-\frac{b}{P\left(\tau_{3}^{2}\right)}\right) ; \\
& S\left(\tau_{3}^{2}\right)=(a+b)(b+\rho)\left[\frac{1}{z_{1} z_{2}}+\tau_{3}^{2}\right]-\frac{A\left(\tau_{3}^{2}\right) P\left(\tau_{3}^{2}\right)}{2(b+\rho)\left(1-\tau_{3}^{2}\right)}\left[\left(z_{1} z_{2}-\frac{b}{P}\right)+\left(z_{1}^{2} z_{2}^{2}+\frac{b}{P}\left[z_{1} z_{2}-\frac{2 B}{A}\right]\right) \tau_{3}^{2}\right] ; \\
& A\left(\tau_{3}^{2}\right)=2\left[k+l\left(1-\tau_{3}^{2}\right)-m\left(1-\tau_{3}^{2}\right)^{2}\right] ; \\
& B\left(\tau_{3}^{2}\right)=2 k+l\left(1-\tau_{3}^{2}\right) ; \\
& \mathrm{C} 0 n\left(\tau_{3}^{2}\right)=b+\rho\left(1-\tau_{3}^{2}\right) .
\end{aligned}
$$

Constants $k, l, m$ are defined by expressions:

$k=(a+2 b)(b+\rho) ; \quad m=(a+b-\rho) \gamma-(\chi+2 \rho)^{2} ;$

$l=(a+2 b) \gamma+(2 b-\chi)(\chi+2 \rho)$.

Here

$$
\begin{aligned}
& a=C_{12} ; \quad b=\frac{1}{2}\left(C_{11}-C_{12}\right)=C_{66} ; \quad \chi=C_{13}-C_{12} ; \\
& \rho=C_{44}-\frac{1}{2}\left(C_{11}-C_{12}\right) ; \quad \rho=C_{44}-\frac{1}{2}\left(C_{11}-C_{12}\right) ; \\
& z_{1} z_{2}=-\sqrt{\frac{2 k}{A\left(\tau_{3}^{2}\right)}} ; \quad z_{1}+z_{2}=i \sqrt{2}\left(\sqrt{\frac{2 k}{A\left(\tau_{3}^{2}\right)}}+\frac{B\left(\tau_{3}^{2}\right)}{A\left(\tau_{3}^{2}\right)}\right)^{1 / 2} .
\end{aligned}
$$




\section{ЭНЕРГИЯ УПРУГОГО ВЗАИМОДЕЙСТВИЯ ТОЧЕЧНЫХ ДЕФЕКТОВ С БАЗИСНОЙ ДИСЛОКАЦИОННОЙ ПЕТЛЕЙ В ЦИРКОНИИ}

О.Г. Троценко, А.В. Бабич

На основе тензорной функции Грина для гексагональных кристаллов, полученной методом ЛифшицаРозенцвейга, посчитано аналитическое выражение энергии упругого взаимодействия радиационных точечных дефектов в модели силовых диполей с дислокационной вакансионной петлей (базисная плоскость залегания $\{0001\}$, вектор Бюргерса $\left.\mathbf{b}^{\text {DS }}=1 / 6\langle 20 \overline{2} 3\rangle\right)$ в цирконии.

\section{ЕНЕРГІЯ ПРУЖНОЇ ВЗАСМОДІЇ ТОЧКОВИХ ДЕФЕКТІВ З БАЗИСНОЮ ДИСЛОКАЦЙНОЮ ПЕТЛЕЮ В ЦИРКОНІЇ}

\section{О.Г. Троценко, А.В. Бабіч}

На основі тензорної функції Гріна для гексагональних кристалів, одержаної методом ЛіфшицяРозенцвейга, пораховано аналітичний вираз енергії пружної взаємодії радіаційних точкових дефектів у моделі силових диполів з дислокаційною вакансійною петлею (базисна площина залягання $\{0001\}$, вектор Бюргерса $\left.\mathbf{b}^{\text {DS }}=1 / 6\langle 20 \overline{2} 3\rangle\right)$ у цирконії. 